\title{
Comparative analysis of the beneficial effects of treadmill training and electroacupuncture in a rat model of neonatal hypoxia-ischemia
}

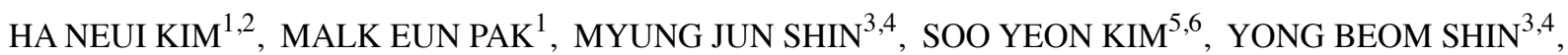 \\ YOUNG JU YUN ${ }^{7}$, HWA KYOUNG SHIN ${ }^{1,2,8}$ and BYUNG TAE CHOI ${ }^{1,2,8}$ \\ ${ }^{1}$ Department of Korean Medical Science, School of Korean Medicine, Pusan National University; \\ ${ }^{2}$ Korean Medical Science Research Center for Healthy-Aging, Pusan National University, Yangsan, \\ Gyeongsangnam-do 50612; ${ }^{3}$ Department of Rehabilitation Medicine, School of Medicine, Pusan National University; \\ ${ }^{4}$ Biomedical Research Institute, Pusan National University Hospital, Busan 49241; ${ }^{5}$ Department of Rehabilitation Medicine \\ and ${ }^{6}$ Research Institute for Convergence of Biomedical Science and Technology, Pusan National University, Yangsan Hospital; \\ ${ }^{7}$ Department of Integrative Medicine, School of Korean Medicine, Pusan National University; ${ }^{8}$ Division of Meridian \\ and Structural Medicine, School of Korean Medicine, Pusan National University, \\ Yangsan, Gyeongsangnam-do 50612, Republic of Korea
}

Received March 21, 2016; Accepted April 19, 2017

DOI: $10.3892 /$ ijmm.2017.2970

\begin{abstract}
In the present study, we investigated whether treadmill training and electroacupuncture (EA) have autonomous or synergistic beneficial effects on deficits caused by neonatal hypoxia-ischemia in Sprague-Dawley rats. For this purpose, rats subjected to hypoxia-ischemia underwent treadmill training and EA stimulation from 4 to 8 weeks of age. Conventional EA (CEA) and scalp EA (SEA) were delivered by electrical stimulation $(2 \mathrm{~Hz}, 1 \mathrm{~mA})$ at traditional acupoints and at the scalp to the primary motor area, respectively. In the behavioral examination, markedly improved performances in the rotarod test were observed in the rats that underwent treadmill exercise, and in the rats that underwent treadmill exercise and CEA compared to the untreated rats subjected to hypoxia-ischemia. An improvement was also observed in the passive avoidance test in the rats that underwent treadmill training and EA. As shown by western blot analysis, the expression levels of neuronal nuclei (NeuN), 2',3'-cyclic-nucleotide 3'-phosphodiesterase and myelin basic protein (MBP) exhibited a significant decrease in the contralateral subventricular zone (SVZ) of the rats subjected to hypoxia-ischemia compared to the controls; however, these expression levels increased following treadmill exercise and
\end{abstract}

Correspondence to: Dr Byung Tae Choi, Department of Korean Medical Science, School of Korean Medicine, Pusan National University, Yangsan, Pusandaehak Street 49, Gyeongsangnam-do 50612, Republic of Korea

E-mail: choibt@pusan.ac.kr

Key words: treadmill, electroacupuncture, cerebral palsy, functional recovery
EA stimulation. As shown by immunohistochemical analyses, the thickness of the corpus callosum and the integrated optical density (IOD) of MBP were significantly increased in the rats subjected to treadmill exercise and EA compared to the untreated rats subjected to hypoxiaa-ischemia. The synergistic effects of treadmill training and EA were also observed in the protein levels and IOD of MBP. A marked increase in the number of bromodeoxyuridine (BrdU)- and BrdU/NeuNpositive cells in the contralateral SVZ was also observed in the rats that underwent treadmill training and EA; the number of BrdU-positive cells was synergistically affected by treadmill training and EA. These results suggest that treadmill training and EA stimulation contribute to the enhancement of behavioral recovery following hypoxia-ischemia via the upregulation of myelin components and neurogenesis. Thus, treatment with EA stimulation, as well as treadmill training offers another treatment option to promote functional recovery in cerebral palsy.

\section{Introduction}

Hypoxia induced by immature lung development is a common pathogenesis underlying premature birth, which has a negative impact on the structural and functional integrity of the brain $(1,2)$. Perinatal hypoxic-ischemic brain injury is associated with the subsequent development of cerebral palsy (CP) $(3,4)$. $\mathrm{CP}$ is associated with profound and life-long chronic neurological disabilities, such as spasticity, motor abnormalities and impairments in learning and memory during growth and adulthood $(3,5,6)$. As there is no definite treatment for $\mathrm{CP}$, different therapeutic strategies have continuously been investigated to improved therapeutic outcomes (7).

Locomotor training, such as treadmill training, is an activitybased strategy used for the promotion of the movement of the limbs and trunk to improve motor and ambulatory skills in chil- 
dren with $\mathrm{CP}$ (8-10). Clinical investigations have indicated that treadmill training improves gross motor function in patients with CP (11-13). Better physical conditioning through activity leads to better health and the prevention of secondary impairments $(7,14)$. Thus, treadmill training is a frequently used rehabilitation model for CP (15). Electroacupuncture (EA), an engrafted electric stimulation, is widely used clinically in many diseases in Oriental medicine, and is accepted as a complementary therapy for the treatment of post-stroke rehabilitation and CP (16-18). EA stimulation induces the differential regulation of genes related to neurogenesis, and enhances cell proliferation and differentiation in the neurogenic area under post-ischemic conditions, which is associated with improved brain function $(19,20)$.

$\mathrm{CP}$ is a non-progressive demyelinating disorder, and white matter injury is the leading cause of this disease in prematurely-born children $(21,22)$. Cerebral white matter injury occurs through the loss of oligodendrocytes, causing damage to myelin and the disruption of nerve conduction (21). Although multifactorial pathophysiology is observed in perinatal brain damage in experimental models, the maturational stage of the brain at the time of injury is a key factor in the pattern of brain recovery (23). Brain injury associated with neonatal hypoxia-ischemia generally coincides with a time window of high susceptibility of neuronal progenitors to excitotoxicity, oxidative stress and inflammation (23-25). Arrest in the differentiation of neuronal progenitors predisposes the brain to defective white-matter tract development in CP $(23,26)$.

Due to the reduced activity, children with a physical disability may not benefit from the effects of treadmill training on brain health (27). The curative effects of acupuncture combined with rehabilitation training has been shown to be significantly more effective than rehabilitation training alone (18). Thus, EA may be employed as an adjuvant therapy for CP. The improvement of behavior by treadmill training or EA has been used in the management of $\mathrm{CP}$; however, the beneficial effects of these treatments on white matter loss and neurogenesis, particularly in EA, are not yet well understood. Rodents exposed to hypoxiaischemia (HI), known as the Vannucci model, have been shown to exhibit motor and cognitive deficits that continue into adulthood $(28,29)$. Functional recovery with the restoration of white matter following HI injury can potentially be induced by the stimulation of endogenous neuronal progenitor cells (30-32).

We thus hypothesized that treadmill training and EA may promote the recovery of behavioral function in a $\mathrm{CP}$-like model via the restoration of white matter and the stimulation of neurogenesis. Thus, in this study, we investigated the effects of these treatments and their combined beneficial effects on deficits consequent to neonatal HI. We aimed to examine whether treadmill training and EA exert beneficial effects against HI-related behavioral deficits via the activation of the myelin component and neuronal progenitor cells. In order to understand currently used treadmill and EA treatments in $\mathrm{CP}$, we performed behavioral testing for motor coordination and memory, and neurogenesis and its related proteins, which are considered markers of brain damage associated with $\mathrm{HI}$ insults.

\section{Materials and methods}

Establishment of the CP-like animal model. Pregnant Sprague-Dawley $(\mathrm{SD})$ rats $(\mathrm{n}=8)$ at embryonic day E17 were obtained from Dooyeol Biotech (Seoul, Korea), housed at $22^{\circ} \mathrm{C}$ under an alternating 12-h light-dark cycle, fed a commercial diet and provided with tap water ad libitum throughout the study period. All experiments were carried out in strict accordance with the recommendations in the Guide for the Care and Use of Laboratory Animals of the National Institutes of Health. The study was approved by the Institutional Animal Care and Use Committee (IACUC) of Pusan National University (approval no. PNU-2015-0771). The establishment of the CP-like animal model was carried out as previously described, with modifications (33). After the rats gave birth, we selected 56, 7-day-old rats for the control and the HI Vannucci model groups. Briefly, on postnatal day $\mathrm{P} 7$, the left common carotid artery of the rats was ligated, and the incision was then sutured. All treatments were administered under $2.0 \%$ isoflurane (Choongwae, Seoul, Korea) anesthesia to minimize animal suffering, using a model VIP 3000 calibrated vaporizer (Midmark, Orchard Park, OH, USA). After surgery, the pups were returned to the mother for recovery, and then placed in a humidified hypoxic chamber with an $8 \% \mathrm{O}_{2} / \mathrm{N}_{2}$ mixture for $3 \mathrm{~h}$, in an incubator maintained at $36^{\circ} \mathrm{C}$. Following the induction of HI, the pups were returned to their cages and remained with their mother. We assessed and monitored the condition of the pups post-surgery daily for any signs of decreased activity, a decrease in food intake and isolation from the littermates. We did not observe any signs of these conditions. The rats were weaned on P21 and randomly divided into 7 groups [control, HI, treadmill (TM), conventional EA (CEA), scalp EA (SEA), TM + CEA and TM + SEA]. Each group consisted of 8 rats. HI-induced injury (signs of $\mathrm{CP}$ ) was examined at $\mathrm{P} 22$ by the cylinder test, as rodents with brain damage have been reported to exhibit behavioral impairment beginning from this time point (29).

Treadmill training. The rats were trained on a treadmill from P22 to the 8th week after birth. During the first week, the rats ran at a speed of $5 \mathrm{~m} / \mathrm{min}$ for $10 \mathrm{~min}$ on the 1st day, gradually progressing to $15 \mathrm{~min}$ on the 5 th day, and this speed and duration were maintained until the end of the 2 nd week. Over the next 3 weeks, the rats ran at $5 \mathrm{~m} / \mathrm{min}$ for $5 \mathrm{~min}$ as a warm-up, and then at an increased speed of up to $7 \mathrm{~m} / \mathrm{min}$ for $15 \mathrm{~min}$, with a recovery period of $5 \mathrm{~m} / \mathrm{min}$ for $5 \mathrm{~min}$.

EA stimulation. The rats were treated with light gaseous anesthesia (1.0\% isoflurane in air), and 2 stainless steel $0.2 \mathrm{~mm}$-diameter needles were inserted. CEA was applied at acupoints corresponding to Baihui (GV20) and right Zusanli (ST36), and SEA was applied bilaterally at subcutaneous tissues of the scalp to the primary motor area. Stimulation with EA was performed with $2 \mathrm{~Hz}$ stimulation $(1 \mathrm{~mA})$ for $20 \mathrm{~min}$, 3 times a week for 5 weeks from P22, using a Pulsemaster Multichannel Stimulator SYS-A300 electrical stimulator (World Precision Instruments, Berlin, Germany). The rats not treated with EA received light gaseous anesthesia (1.0\% isoflurane for $20 \mathrm{~min}$ ).

Bromodeoxyuridine (BrdU) labeling. BrdU (B5002-5G; Sigma-Aldrich; Merck KGaA, Darmstadt, Germany) is a synthetic thymidine analog that becomes incorporated into a cell's DNA when the cell is dividing during the $S$ phase of the cell cycle. For the labeling of proliferating cells in the subven- 
tricular zone (SVZ) of the brain, the rats were injected with BrdU (50 mg/kg, i.p.) once daily for 2 weeks from P29. The effect of treadmill exercise and EA on the subsequent survival of the newborn cells was determined at the end of the study period.

\section{Behavioral assessments}

Open-field test. The open-field apparatus consisted of a black box $(30 \times 30 \times 40 \mathrm{~cm})$. After 5 min of adaptation in the black box, measurements were performed for $5 \mathrm{~min}$ as the total distance traveled by placing the rats for the evaluation of locomotor activity. Results of the experiment were recorded using SMART 2.5.18 software (Panlab, S.L.U., Barcelona, Spain).

Cylinder test. The cylinder test was performed for the measurement of forelimb use for the evaluation of functional recovery from the unilateral brain lesion. The rat was placed in a glass cylinder, $20 \mathrm{~cm}$ in diameter and $30 \mathrm{~cm}$ in height, and the number of supports in the wall were counted with the ipsilateral (to the lesion) forelimb and the contralateral forelimb support with both forelimbs against the wall and recorded during lateral exploration. Total count score $=($ both hands movement - ipsilateral forelimb movement)/total of 20 contacts.

Accelerated rotarod test. The rotarod test was performed using a rotarod apparatus (Panlab S.L.U.) to evaluate motor balance and coordination following HI. After 3 adaptation trials, each rat was placed on the rotating rod for 3 trials per day at a speed of $4 \mathrm{rpm}$, which was gradually increased to $40 \mathrm{rpm}$ in $5 \mathrm{~min}$. The time that an animal was able to hold itself on the rod was recorded as the latency to fall.

Hanging wire grip test. The hanging wire grip test was performed for the evaluation of the grasping ability for forelimb strength and coordination of movement. The rats used their forelimbs to suspend their bodies from a wire $0.3 \mathrm{~cm}$ in diameter, $40 \mathrm{~cm}$ in length and $45 \mathrm{~cm}$ above soft ground. The time spent holding the wire was recorded during 3 daily trials for each rat. A maximum cut-off latency time of $60 \mathrm{sec}$ was recorded.

Gait analysis. CatWalk (Noldus Information Technology, Wageningen, The Netherlands), a quantitative gait analysis system, was used for gait analysis. Before recording, the test course was pre-run with an animal to induce a non-stressful environment, and 3 trials were performed once a week. The steps were automatically labeled as right forepaw, right hind paw, left forepaw and left hind paw. Animals that failed to complete a run within $8 \mathrm{sec}$ were allowed an additional re-run. In addition, individual footprint parameters, such as the stride length and swing speed were also analyzed automatically after identification of individual footprints.

Passive avoidance test. The passive avoidance test was used for the assessment of cognitive function and memory, after an injury model using a passive avoidance task (Med Associates Inc., St. Albans, VT, USA). After 1 day of adaptation in the passive avoidance case, the rat was placed in an illuminated compartment for the learning trial. If it crossed the door into the dark compartment, which then closed automatically, a single inescapable scrambled foot shock $(0.8 \mathrm{~mA}, 3 \mathrm{sec})$ was delivered through the grid base. In this study, the procedure was repeated until the latency to enter the dark box was longer than $180 \mathrm{sec}$. The retention test was performed $24 \mathrm{~h}$ after the learning trial in the same manner. Animals that did not enter the dark chamber during the retention test were allotted a latency of $180 \mathrm{sec}$.

Western blot analysis. After the behavioral test, the rats were anesthetized with $8 \%$ chloral hydrate $(50 \mathrm{mg} / \mathrm{kg}, \mathrm{C} 8383-100 \mathrm{G}$; Sigma-Aldrich; Merck KGaA) and sacrificed via intracardial perfusion with saline. Each collected brain tissue in the SVZ was washed in cold HEPES buffer and homogenized in lysis buffer [200 mM Tris (pH 8.0), $150 \mathrm{mM} \mathrm{NaCl,} 2 \mathrm{mM}$ EDTA, $1 \mathrm{mM} \mathrm{NaF}, 1 \%$ NP-40, $1 \mathrm{mM}$ phenylmethanesulfonyl fluoride, $1 \mathrm{mM} \mathrm{Na} \mathrm{VO}_{4}$ and protease inhibitor cocktail]. Equal amounts of proteins were then separated by $5-10 \%$ sodium dodecyl sulfate-polyacrylamide gel electrophoresis, followed by the transfer of resolved proteins onto nitrocellulose membranes (Whatman, Dassel, Germany). After transfer, the membranes were incubated with 5\% skim milk in PBST for $1 \mathrm{~h}$, followed by incubation overnight at $4^{\circ} \mathrm{C}$ with the following primary antibodies: neuronal nuclei (NeuN; MAB377; EMD; Millipore, Billerica, MA, USA), doublecortin (DCX; sc-8066; Santa Cruz Biotechnology, Inc., Santa Cruz, CA, USA), 2',3'-cyclic-nucleotide 3'-phosphodiesterase (CNPase; ab6319; Abcam, Cambridge, UK), NG2 (MAB5384; Chemicon International, Inc., Billerica, MA, USA), myelin basic protein (MBP; ab40390; Abcam), glial fibrillary acidic protein (GFAP; MAB360; EMD; Millipore) and Iba-1 (016-20001; Wako Pure Chemical Industries, Ltd., Osaka, Japan). The membranes were then incubated for $1 \mathrm{~h}$ at room temperature with secondary antibodies according to the species for each antibody host against goat-anti-mouse (sc-2005), goat anti-rabbit (sc-2004) and donkey anti-goat (sc-2020) (all from Santa Cruz Biotechnology, Inc.). $\beta$-actin was used as a loading control for all the experiments. The quantification of immunoreactivity corresponding to the bands was performed by densitometric analysis using ImageQuant LAS 4000 software (Fujifilm, Tokyo, Japan).

Immunohistochemistry. After behavioral assessments, the rats anesthetized with isoflurane received intracardial perfusion with saline, followed by $4 \%$ paraformaldehyde in PBS. The brains were removed, post-fixed in the same fixative for $4 \mathrm{~h}$ at $4^{\circ} \mathrm{C}$, and immersed in $30 \%$ sucrose for $48 \mathrm{~h}$ for cryoprotection. Frozen $20 \mu \mathrm{m}$-thick sections from bregma 0 to $+1 \mathrm{~mm}$ were incubated for post-fixation with formaldehyde, and blocked with a blocking buffer (1X PBS/5\% normal goat serum $/ 0.3 \%$ Triton $\mathrm{X}-100$ ) for $1 \mathrm{~h}$ at room temperature. The sections were incubated with the following primary antibodies to BrdU (MCA2483, AbD Serotec, Hercules, CA, USA), NeuN (ABN78, EMD; Millipore) and MBP (ab40390; Abcam) overnight in PBS at $4^{\circ} \mathrm{C}$. After washing with PBS, the sections were incubated with the fluorescent secondary antibodies against horse anti-mouse (FI-2000), goat anti-rabbit (TI-1000) and goat anti-rabbit (FI-1000) (all from Vector Laboratories, Inc., Burlingame, CA, USA) for $2 \mathrm{~h}$ at room temperature in the dark, and then washed 3 times with PBS. Subsequently, slides were mounted in mounting medium (Dako, Glostrup, Denmark) and captured using a fluorescence microscope (Carl Zeiss Imager M1; Carl Zeiss, Inc., Gottingen, Germany). IMT i-Solution (IMT i-Solution Inc., Burnaby, $\mathrm{BC}$, Canada) was used for the automatic measurement of cell counting, thickness and integrated optical density (IOD). 


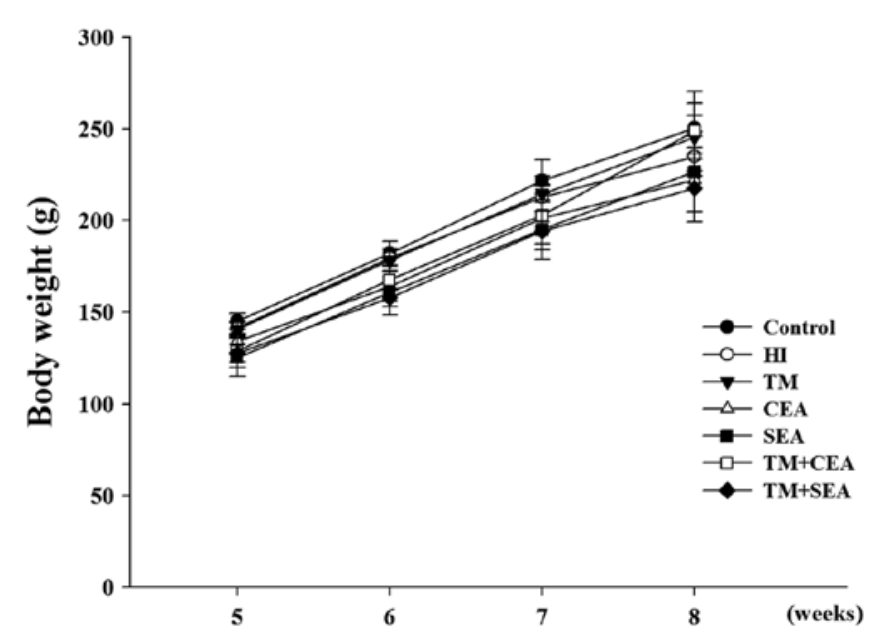

Figure 1. Changes in body weight with age. There were no significant differences among all rat groups $(\mathrm{n}=8)$.

Statistical analysis. All data are expressed as the means \pm SEM, and analysis of variance (ANOVA) was performed using the SigmaStat statistical program version 11.2 (Systat Software, Inc., San Jose, CA, USA). Statistical analysis of the data was performed using one-way ANOVA via Tukey's post hoc comparison when comparing more than 2 groups. A alue of $\mathrm{P}<0.05$ was considered to indicate a statistically significant difference.

\section{Results}

Behavioral analysis for functional recovery. Behavioral analysis was performed to determine the potential benefits of treadmill training and EA. Body weight increased with age, but no significant differences were detected between the rats (Fig. 1). Behavioral examination included tests for the assessment of sensorimotor recovery (cylinder test), locomotor activity (openfield test), gait analysis (CatWalk), motor coordination (rotarod and hanging wire grid test) and memory (passive avoidance test). Behavioral deficits in locomotor activity and gait analysis did not exhibit significant differences between the control and HI group rats (data not shown); however, the tests for sensorimotor recovery, motor coordination and memory revealed significant changes (Fig. 2). For the sensorimotor recovery from unilateral brain lesion assessed by the cylinder test, the numbers of cylinder wall contacts with impaired forelimb increased in the rats that underwent with treadmill training or EA. Treadmill training and co-treatment (treadmill training and EA) led to a greater improvement in the use of the forelimb than CEA and SEA alone; however, there were no significant differences between the HI group rats and those that underwent treadmill training or EA (Fig. 2A).

In the assessment of motor coordination by the rotarod and hanging wire grid test, a significant improvement in the latency time in performance was observed in the rotarod test at 8 weeks, and from 6 to 8 weeks in the rats that underwent treadmill training, or co-treatment (treadmill training and CEA), respectively (Fig. 2B). In the hanging wire grid test, the rats that underwent treadmill training and co-treatment (treadmill training and EA) exhibited a longer time of holding the bar than the HI group rats, although there were no significant differences (Fig. 2C).

In the assessment of memory capabilities by the passive avoidance test, the degree of impairment in memory was dependent on the HI condition, and a significant increase in retention latency was observed in the rats that underwent treadmill training or EA, and in those that underwent both treadmill training and EA compared to the HI group rats (Fig. 2D). All these results suggest that treadmill training and EA stimulation exert significant beneficial effects, such as sensorimotor recovery, motor coordination and memory capabilities in rats subjected to HI; the synergistic effects of treadmill training and EA were also observed in motor coordination.

Western blot analysis for the expression of brain recovery markers. To investigate whether functional recovery occurs through expression at the protein level, western blot analysis was performed for the expression of brain recovery markers. As regards the expression of cell components and its proliferative markers in the brain, the expression of NeuN, CNPase and MBP in the HI group rats was significantly decreased compared to the control rats (Figs. 3 and 4). However, the expression of $\mathrm{NeuN}$ was significantly higher in the rats that underwent treadmill training and EA compared to the HI group rats (Figs. 3B and 4B). The expression of CNPase exhibited a significant increase in the rats that underwent treadmill training and EA, except in the rats treated with CEA alone compared to the HI group rats, and a significantly higher expression was also observed in the rats treated with SEA alone or co-treated (treadmill training and SEA) compared to the rats that underwent treadmill training alone (Figs. 3C and 4C). A markedly higher expression of MBP was observed in the rats that underwent treadmill training and EA compared to the HI group rats. A markedly higher expression of MBP was also observed in the rats that underwent both treadmill training and EA compared to the rats that underwent treadmill training alone (Figs. 3D and 4D). These results suggest that treadmill training and EA promote functional recovery following HI-induced perinatal brain injury, by increasing the expression of key proteins, including myelin components.

Immunohistochemical analysis for myelin components and neurogenesis. To examine myelin components, we measured the IOD of MBP staining and the thickness of the corpus callosum. The IOD of MBP staining and the thickness of the corpus callosum were significantly decreased in the HI group rats compared to the control rats. These changes were significantly attenuated in the rats that underwent treadmill training and EA compared to the HI group rats, and greater changes in the IOD of MBP were also observed in the rats that underwent both treadmill training with EA compared to the rats that underwent treadmill training alone (Fig. 5). To investigate the effects of treadmill training and EA on the proliferation and differentiation of neural progenitor cells, we examined positive cells for BrdU and BrdU/NeuN in the SVZ of the contralateral hemisphere. A significant increase in the number of BrdU-positive cells was observed in the SVZ of the HI group rats compared to the controls, but a limited number of cells exhibited a BrdU/NeuN double-positive reaction. The rats that undewent treadmill training and those that underwent both 

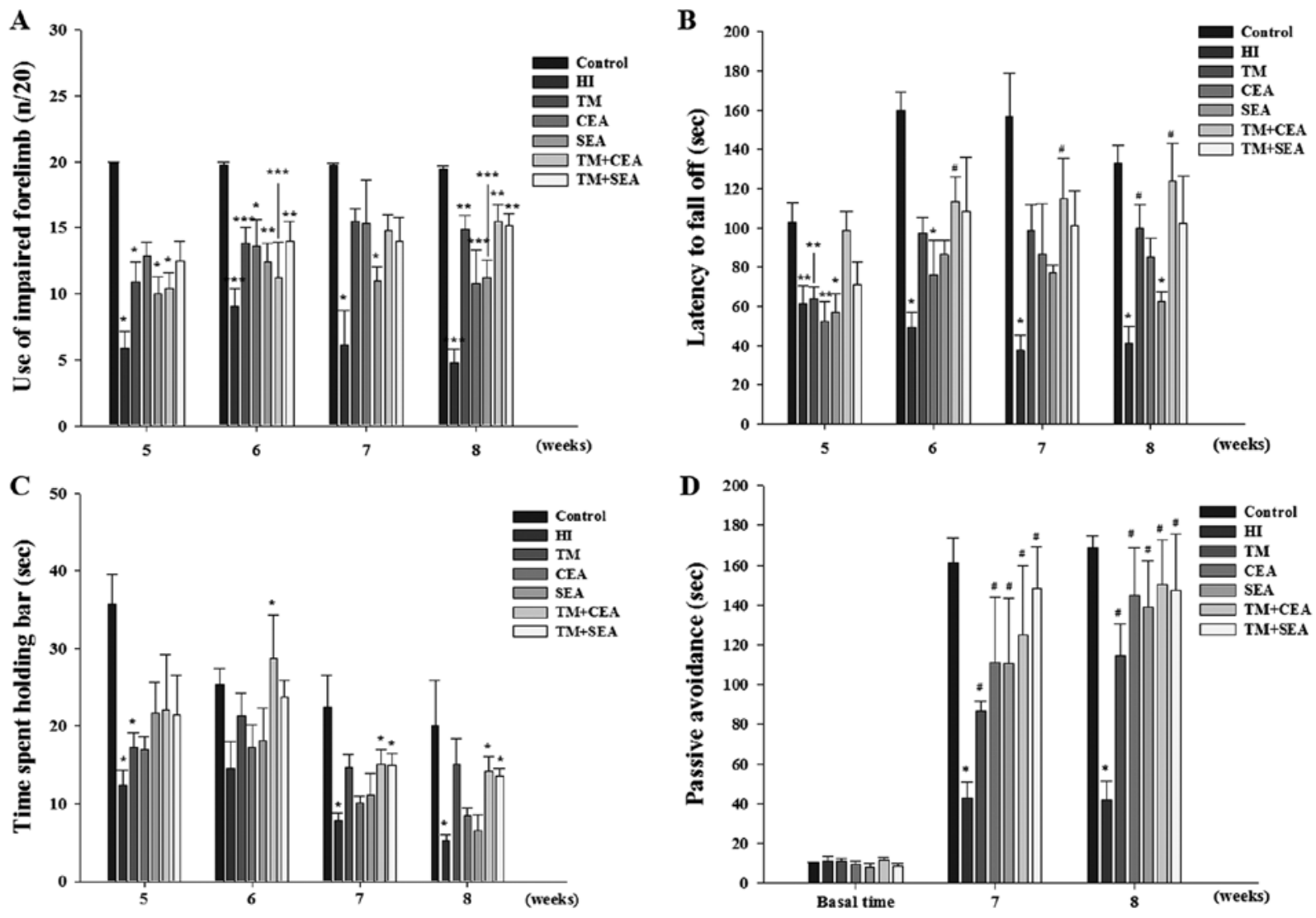

Figure 2. Behavioral tests for the assessment of functional deficits caused by hypoxia-ischemia (HI). (A) Cylinder, (B) accelerated rotarod, (C) hanging wire grip and (D) passive avoidance tests were performed and compared with each group $(n=8)$. HI group rats exhibited severe functional deficits compared to the control rats, and deficits in accelerated rotarod and passive avoidance test were significantly attenuated by treadmill training and electroacupuncture (EA) treatment. Data are the means \pm SEM. ${ }^{*} \mathrm{P}<0.05,{ }^{* *} \mathrm{P}<0.01$ and ${ }^{* * * *} \mathrm{P}<0.001$ vs. control rats; ${ }^{*} \mathrm{P}<0.05$ vs. HI group rats.

A

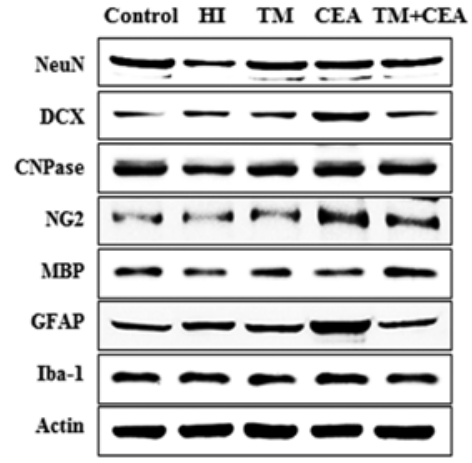

C

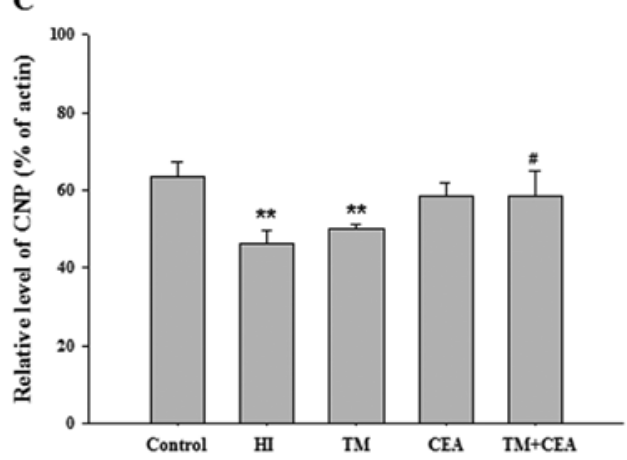

B

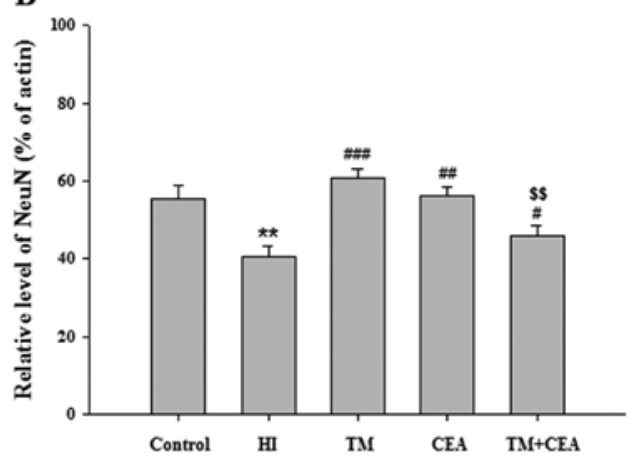

D

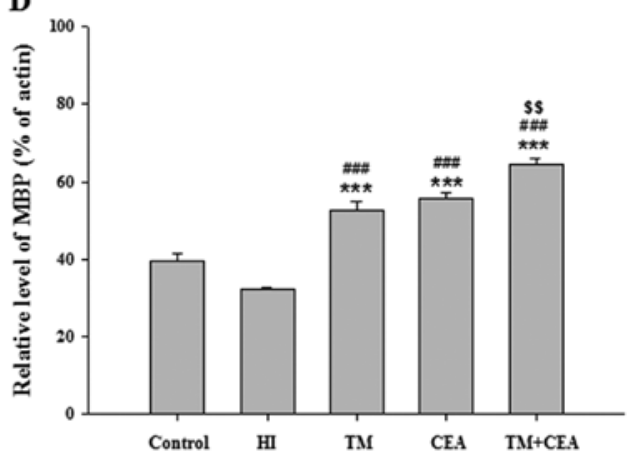

Figure 3. Western blot analysis and densitometric analysis for functional recovery markers in contralateral subventricular zone (SVZ) of the brain in rats that underwent treadmill training and conventional electroacupuncture (CEA). (A) Typical result from 3 independent experiments $(\mathrm{n}=6)$. The expression of (B) neuronal nuclei (NeuN), (C) 2',3'-cyclic-nucleotide 3'-phosphodiesterase (CNPase) and (D) myelin basic protein (MBP) in rats subjected to hypoxia-ischemia (HI) was significantly increased by treadmill training and CEA. ${ }^{* *} \mathrm{P}<0.01$ and ${ }^{* * *} \mathrm{P}<0.001$ vs. control rats; ${ }^{*} \mathrm{P}<0.05,{ }^{\# \#} \mathrm{P}<0.01$ and ${ }^{\# \# \#} \mathrm{P}<0.001$ vs. HI group rats; ${ }^{\$ \$} \mathrm{P}<0.01$ vs. rats that underwent treadmill training alone. 
A

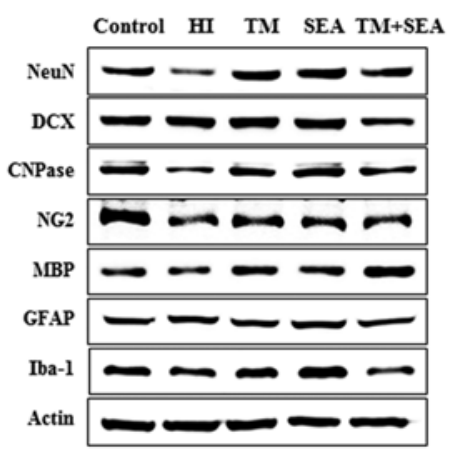

C

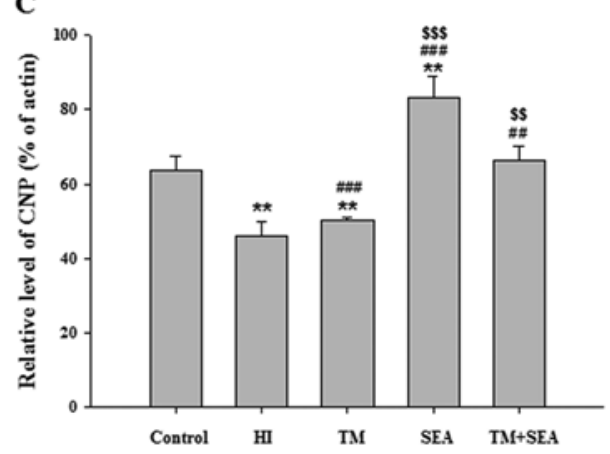

B

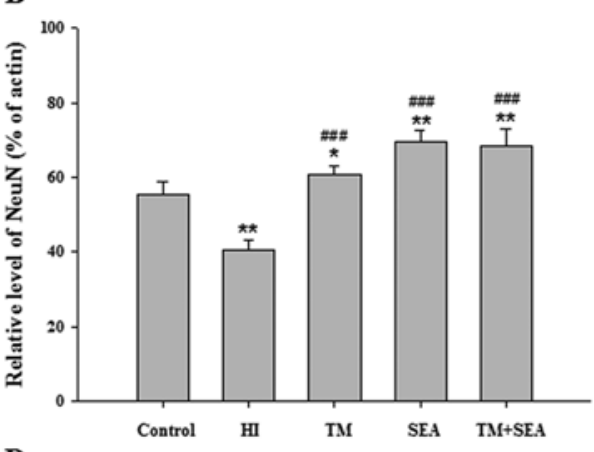

D

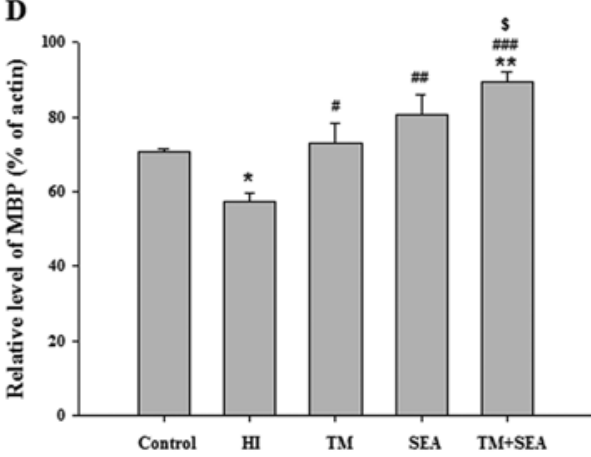

Figure 4. Western blot analysis and densitometric analysis for functional recovery markers in contralateral subventricular zone (SVZ) of the brain in rats that underwent treadmill training and scalp electroacupuncture (SEA). (A) Typical result from 3 independent experiments $(\mathrm{n}=6)$. The expression of $(\mathrm{B})$ neuronal nuclei (NeuN), (C) 2',3'-cyclic-nucleotide 3'-phosphodiesterase (CNPase) and (D) myelin basic protein (MBP) in rats subjected to hypoxia-ischemia (HI) was significantly increased by treadmill training and SEA. ${ }^{*} \mathrm{P}<0.05$ and ${ }^{* *} \mathrm{P}<0.01$ vs. control rats; ${ }^{\#} \mathrm{P}<0.05,{ }^{\# \#} \mathrm{P}<0.01$ and ${ }^{\# \# "} \mathrm{P}<0.001$ vs. HI group rats; ${ }^{\$} \mathrm{P}<0.05$, ${ }^{\$ \$} \mathrm{P}<0.01$ and ${ }^{\$ \$ \$} \mathrm{P}<0.001$ vs. rats that underwent treadmill training alone.

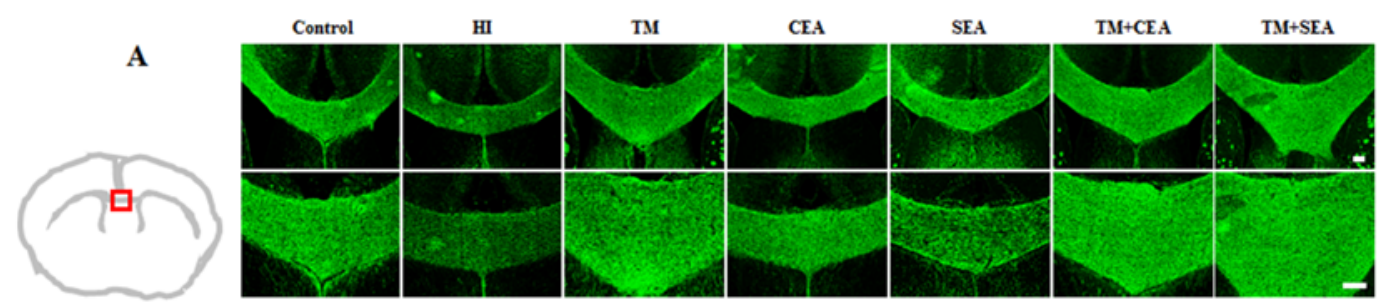

B

C
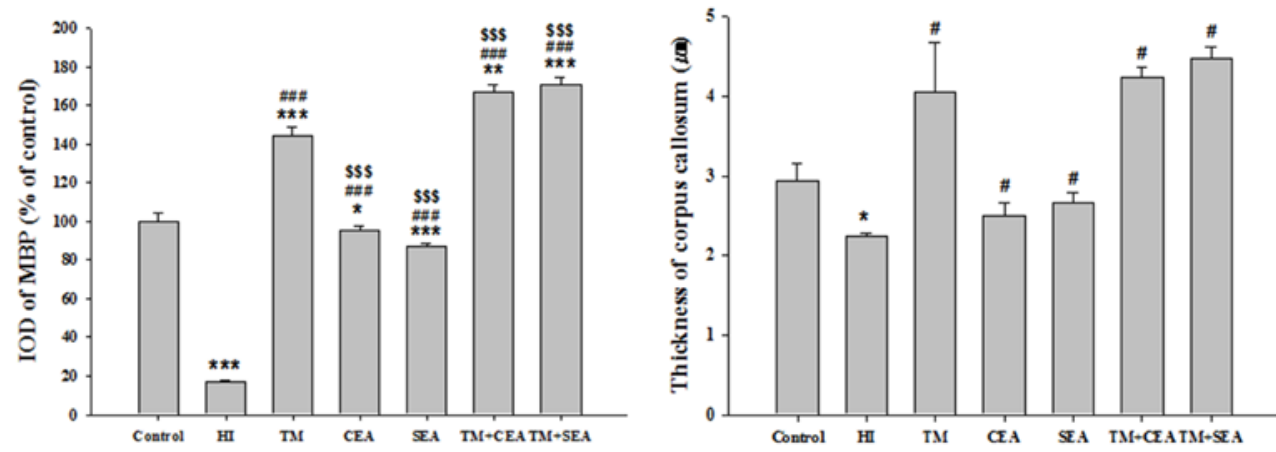

Figure 5. Myelin components analysis in the corpus callosum. (A) Photomicrograph and (B) its histogram for integrated optical density (IOD) of myelin basic protein (MBP) and (C) thickness of the corpus callosum $(n=6)$. A marked increase in the IOD of MBP and thickness of the corpus callosum were observed in the rats that underwent treadmill training and electroacupuncture (EA) compared to the untreated rats subjected to hypoxia-ischemia $(\mathrm{HI}){ }^{*} \mathrm{P}<0.05$ and ${ }^{* * *} \mathrm{P}<0.001$ vs. control rats; ${ }^{\#} \mathrm{P}<0.05$ and ${ }^{\# \# \#} \mathrm{P}<0.001$ vs. HI rats; ${ }^{\text {s\$s }} \mathrm{P}<0.001$ vs. treadmill alone treated rats. Scale bars, $200 \mu \mathrm{m}$.

treadmill training and EA exhibited a significant increase in the number of BrdU- or BrdU/NeuN-positive cells compared to the HI group rats. A significant number of positive cells for BrdU was also observed in the rats that underwent both treadmill training and EA compared to the rats that undewent treadmill training alone. The number of BrdU/NeuN double-positive cells was also significantly increased in the rats treated with CEA or SEA compared to the HI group rats (Fig. 6). These results 
A
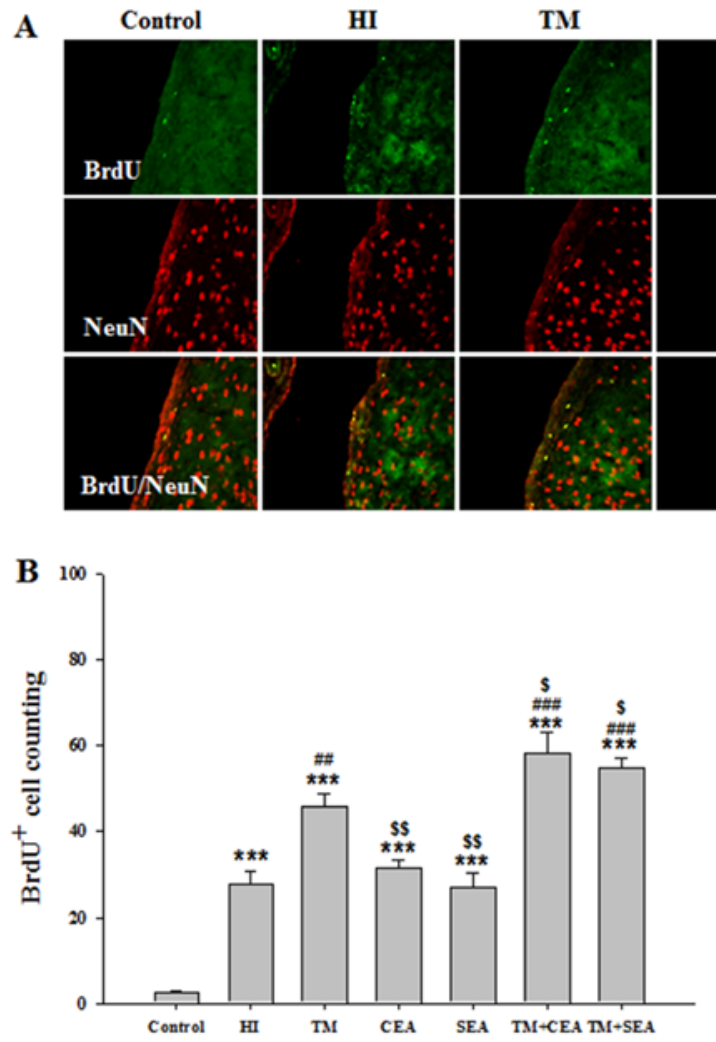

CEA
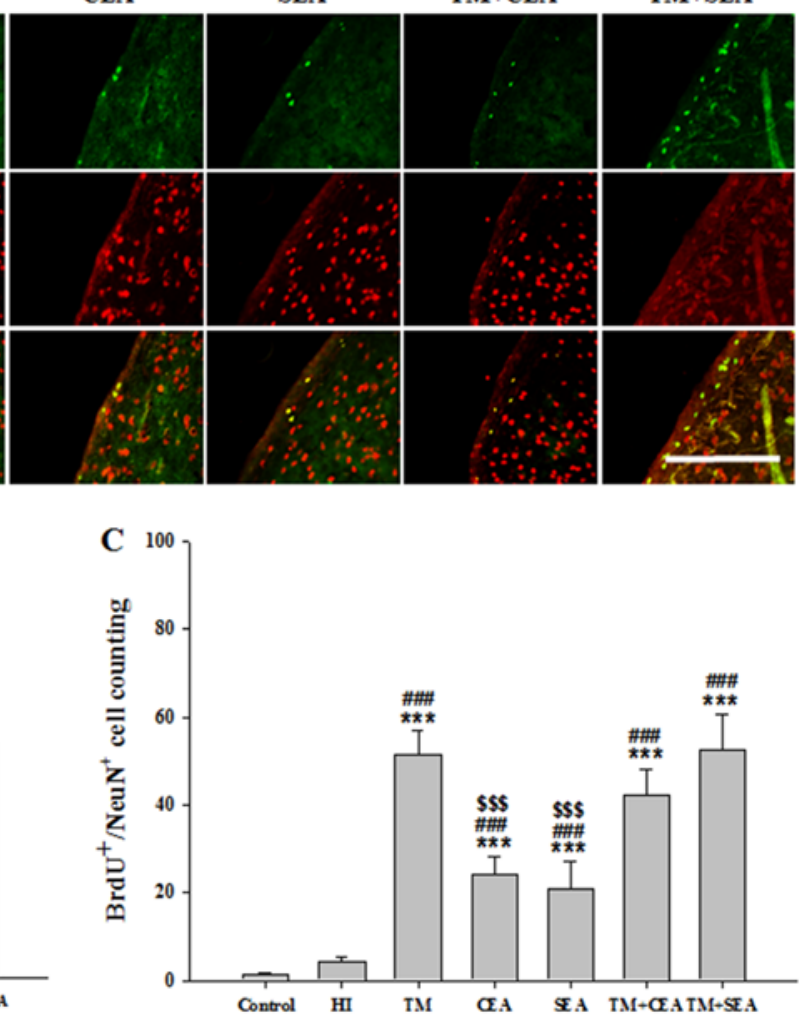

Figure 6. Effects of treadmill training and electroacupuncture (EA) treatment on neurogenesis in the subventricular zone (SVZ) of the brain. (A) Photomicrograph and (B) its histogram for bromodeoxyuridine (BrdU) and (C) BrdU/neuronal nuclei (NeuN)-positive cells ( $\mathrm{n}=6$ ). (B) The numbers of proliferated progenitor cells and differentiated neurons indicated by BrdU- and BrdU/NeuN-positive were significantly increased in the rats that underwent treadmill training and EA compared to the untreated rats that were subjected to hypoxia-ischemia $(\mathrm{HI}) .{ }^{* * *} \mathrm{P}<0.001$ vs. control rats; ${ }^{\# \#} \mathrm{P}<0.01$ and ${ }^{\# \# \#} \mathrm{P}<0.001$ vs. $\mathrm{HI}$ rats; ${ }^{\$} \mathrm{P}<0.05,{ }^{\$ \$} \mathrm{P}<0.01$ and ${ }^{\$ \$} \mathrm{P}<0.001$ vs. treadmill alone treated rats. Scale bars, $200 \mu \mathrm{m}$.

suggest that treadmill training and EA stimulation significantly attenuate white matter injury in the corpus callosum following HI insults, and enhance the number of newly formed neuroblasts and differentiation to mature neurons in the SVZ.

\section{Discussion}

In this study, we found that EA, as well as treadmill training exerted autonomous and synergistic beneficial effects on behavioral deficits of motor coordination and memory in a rat CP-like model subjected to HI. Treadmill training and EA in this animal model were effective in ameliorating behavioral deficits by enhancing myelin components and the proliferation and differentiation of neuronal progenitor cells. Thus, with treadmill training, the clinical benefits of EA may be another approach for the treatment of motor deficits, as well as memory impairments in CP. Our study provides new insight into the therapeutic treatment of CP.

Pre-term births have increased, and continue to rise steadily, due to the delivery by mothers of a more advanced age, fertility treatments and environmental factors (2). Perinatal HI in preterm births remains a major cause of $\mathrm{CP}$ and is associated with a life-long impact on motor abnormalities and cognitive deficits lasting until adolescence $(1,3,4,6)$. Treadmill training for $\mathrm{CP}$, both full weight-bearing and partial weight-bearing, is a strategy for locomotor training based on principles that promote the movement of the limbs and trunk to generate sensory information (10). This training can improve walking speed and gross motor function in patients with neuromuscular disorders, such as CP $(12,13,15)$.

EA therapy can ameliorate both the motor deficits and cognitive impairments in development of HI, including CP (16-18). Two types of EA were employed in the present study. To obtain therapeutic effects, electrical stimulation of CEA was applied at traditional specific acupoints, and SEA at subcutaneous tissues of the scalp to the primary motor area. SEA therapy is a novel therapeutic EA technique based on modern neuro-anatomical knowledge of the functional localization of the cerebral cortex. We employed SEA as this technique is similar to that for transcranial direct-currents, which involves stimulation over the cerebral cortex with an electrical impulse leading to improved functional performance in CP (34).

As neurological disorders in $\mathrm{CP}$ are characterized by motor abnormalities and cognitive deficits $(1,6,35)$, we first performed behavioral tests for these long-term deficits to compare the outcomes of treadmill training and EA stimulation. We used an HI model that shows motor and cognitive deficits by unilateral carotid artery ligation and hypoxia, whose deficits last well into adulthood $(36,37)$. HI affected behavioral performance with sensorimotor recovery, motor coordination and memory, but treadmill and EA treatment exhibited better behavioral performances, particularly in motor coordination and memory.

In comparing the efficacy between treadmill training and EA, treadmill training and EA exerted beneficial effects for memory capabilities, as shown by the passive avoidance 
test. However, for the assessment of motor coordination by the rotarod test, a significant improvement was observed in the rats that underwent treadmill training, suggesting that treadmill training may mainly compensate for deficits in gross motor coordination and balance from neonatal HI brain injury, via the improvement of muscular fitness as activity-based training $(13,33)$. In addition, CEA treatment combined with treadmill training exerted some synergistic effects compared with rehabilitation training alone as in a previous study (18). However, in this study, the CEA alone-treated rats exhibited a somewhat lesser improvement compared to the rats that underwent treadmill training alone, suggesting that treadmill training may mainly improve motor and ambulatory skills in rats that underwent both treadmill training and CEA.

Next, western blot analysis was performed for cell components of the brain with neuronal progenitor markers, to assess the associations between the expression of these proteins and behavioral recovery $(21,22,38,39)$. The injury in the Vannucci model of HI induces damage restricted to the subcortical and periventricular white matter, cerebral cortex, hippocampus and striatum of the ipsilateral hemisphere $(36,37)$. To determine the beneficial effects of treadmill training and EA, we analyzed the contralateral SVZ to exclude neurodegeneration and white matter loss by carotid artery ligation.

Although markers for astrocytes (GFAP), microglia (Iba-1) and neuronal progenitor cells (DCX) exhibited no significant changes, treadmill training and EA resulted in a significantly increased expression of key proteins for neuron, oligodendroglia and myelin compared to the HI group rats. These findings suggest that perinatal HI alters development through a delay in maturation of affected cell types, including neurons and oligodendroglia $(2,21,22)$. Behavioral deficits are associated with white matter injury, as well as the expression of MBP in white matter $(23,28,29)$. In this study, we measured the thickness and components of myelin in the corpus callosum to confirm the expression of MBP. A markedly increased thickness and density of myelin were observed in rats that underwent treadmill training and EA compared to the HI group rats. These findings suggest that treadmill training and EA may promote functional recovery from HI-induced injury through the promotion of maturation of myelin components of the brain.

The mechanisms through which treadmill training and EA ameliorate motor dysfunction and memory impairment by the restoration of white matter in neonatal HI are not yet clear. A well-studied hypothesis in this regard involves the upregulation of neurotrophins (NTs), which play a role in the evolution of clinical signs of neuronal dysfunction, through physical activity, such as using a treadmill $(27,40)$. As for candidate neurotrophic factors, significantly elevated NTs, such as nerve growth factor, brain-derived neurotrophic factor (BDNF), NT-4 and insulin-like growth factor 1 promote neuronal plasticity and enhance myelin components in a model of motor dysfunction under treadmill training (41-43). Moreover, BDNF is considered a main NT in motosensory and cognitive function response to physical exercise $(43,44)$. It has been suggested that the physiological processes of CEA are similar to those resulting from physical exercise, as CEA innervated nerve fibers respond similarly as to muscle contractions (45). CEA stimulation promotes the functional recovery of motor coordination and memory with the promoted expression of MBP by the increased expression of BDNF, vascular endothelial growth factor (VEGF) and NT-3 or its receptors in a model of ischemia $(46,47)$. To the very best of our knowledge, there are no available published studies to date on the possible mechanisms of SEA; however, a similar treatment, transcranial direct-current stimulation, has been shown to lead to improvements in functional performance, and BDNF plays a role in this stimulation-induced motor cortex plasticity $(34,48)$.

Neurological impairments in endogenous neural progenitor cells were likely improved through the production of new neuronal and glial cells. Neuronal progenitor cells migrate from the SVZ into the damaged striatum or other sites, regions where neurogenesis does not occur (23). Neural progenitor cells can provide an appropriate therapeutic strategy for neurological disorders, but the number of newly born cells is too small for the recovery of neurological functions $(31,49)$. Therefore, the simple proliferation of neural progenitor cells does not guarantee successful recovery from functional impairments. Finally, we addressed the role of SVZ neurogenesis from endogenous progenitor cells, resulting from treatment with treadmill and EA in hypoxia-ischemic rats.

Our study demonstrated that treadmill training, and treadmill training together with EA significantly induced the proliferation of progenitor cells. Treadmill and CEA can lead to the release of NTs and enhance the proliferation of progenitor cells by neurogenesis $(20,27,50,51)$. The lack of progenitor cells, such as the oligodendrocyte lineage, adversely affects neonatal white-matter injury as the underlying cause for functional deficits $(23,47)$. Treadmill exercise induces neurogenesis, requiring signaling through BDNF and VEGF, or its receptor expressions and downstream kinase signaling pathway (52-54). CEA can also promote the proliferation and maturation of oligodendrocytes via NT-3 and -4 signaling (47,51). Therefore, the proliferation and differentiation of progenitor cells by treadmill training and EA positively affects the loss of whitematter with functional deficits in CP (23).

There are no definite available treatments for CP. As shown by the results of our study for motor coordination, the thickness of the corpus callosum and mature neuron from progenitor cells, a significant improvement was observed in the rats that underwent treadmill training alone. Therefore, treadmill training has been used as a rehabilitation model for functional recovery in patients. However, some patients with physical disability are unable to move the limbs and trunk in order to generate sensory information. EA stimulation can be applied for the treatment of patients who are incapable of treadmill training. In the present study, some therapeutic effects were also obtained by electrical stimulation of specific acupoints or scalp of the primary motor area. Consequently, treadmill training and EA stimulation promote the recovery of functions such as sensorimotor deficits, motor coordination and cognitive impairments in HI rats.

Thus, as demonstrated in this study, treadmill training and EA may contribute to each autonomous beneficial effect with some synergistic effects from functional deficits through the upregulation of myelin components and neurogenesis. Thus, treatment with EA stimulation, as well as treadmill training offers another treatment option for the functional recovery 
in $\mathrm{CP}$, and can be applied to the treatment of patients with physical disability.

\section{Acknowledgements}

This study was supported by the Convergence of Conventional Medicine and Traditional Korean Medicine R\&D Project funded by the Ministry of Health and Welfare through the Korea Health Industry Development Institute (KHIDI) (grant no. HI14C0704).

\section{References}

1. Eikenes L, Løhaugen GC, Brubakk AM, Skranes J and Håberg AK: Young adults born preterm with very low birth weight demonstrate widespread white matter alterations on brain DTI. Neuroimage 54: 1774-1785, 2011.

2. Salmaso N, Jablonska B, Scafidi J, Vaccarino FM and Gallo V: Neurobiology of premature brain injury. Nat Neurosci 17: 341-346, 2014.

3. Strata F, Coq JO, Byl N and Merzenich MM: Effects of sensorimotor restriction and anoxia on gait and motor cortex organization: implications for a rodent model of cerebral palsy. Neuroscience 129: 141-156, 2004.

4. Bennet L, Tan S, Van den Heuij L, Derrick M, Groenendaal F, van Bel F, Juul S, Back SA, Northington F, Robertson NJ, et al: Cell therapy for neonatal hypoxia-ischemia and cerebral palsy. Ann Neurol 71: 589-600, 2012.

5. van Handel M, Swaab H, de Vries LS and Jongmans MJ: Long-term cognitive and behavioral consequences of neonatal encephalopathy following perinatal asphyxia: a review. Eur J Pediatr 166: 645-654, 2007.

6. Aarnoudse-Moens CS, Weisglas-Kuperus N, van Goudoever JB and Oosterlaan J: Meta-analysis of neurobehavioral outcomes in very preterm and/or very low birth weight children. Pediatrics 124: 717-728, 2009.

7. Damiano DL: Activity, activity, activity: rethinking our physical therapy approach to cerebral palsy. Phys Ther 86 : 1534-1540, 2006.

8. Richards CLMF, Dumas F, Marcoux S, Lepage C and Menier C: Early and intensive treadmill locomotor training for young children with cerebral palsy: a feasibility study. Pediatr Phys Ther 9: 158-165, 1997.

9. Cherng RJ, Liu CF, Lau TW and Hong RB: Effect of treadmill training with body weight support on gait and gross motor function in children with spastic cerebral palsy. Am J Phys Med Rehabil 86: 548-555, 2007.

10. Tsai SW, Chen HL, Chang YC and Chen CM: Molecular mechanisms of treadmill therapy on neuromuscular atrophy induced via botulinum toxin A. Neural Plast 2013: 593271, 2013.

11. Willoughby KL, Dodd KJ and Shields N: A systematic review of the effectiveness of treadmill training for children with cerebral palsy. Disabil Rehabil 31: 1971-1979, 2009.

12. Mutlu A, Krosschell K and Spira DG: Treadmill training with partial body-weight support in children with cerebral palsy: a systematic review. Dev Med Child Neurol 51: 268-275, 2009.

13. Chrysagis N, Skordilis EK, Stavrou N, Grammatopoulou E and Koutsouki D: The effect of treadmill training on gross motor function and walking speed in ambulatory adolescents with cerebral palsy: a randomized controlled trial. Am J Phys Med Rehabil 91: 747-760, 2012

14. Jones MW, Morgan E, Shelton JE and Thorogood C: Cerebral palsy: introduction and diagnosis (part I). J Pediatr Health Care 21: 146-152, 2007.

15. Wessels M, Lucas C, Eriks I and de Groot S: Body weight-supported gait training for restoration of walking in people with an incomplete spinal cord injury: a systematic review. J Rehabil Med 42: 513-519, 2010.

16. Wu P, Mills E, Moher D and Seely D: Acupuncture in poststroke rehabilitation: a systematic review and meta-analysis of randomized trials. Stroke 41: e171-e179, 2010.

17. Duncan B, Shen K, Zou LP, Han TL, Lu ZL, Zheng H, Walsh M, Venker C, Su Y, Schnyer R, et al: Evaluating intense rehabilitative therapies with and without acupuncture for children with cerebral palsy: a randomized controlled trial. Arch Phys Med Rehabil 93: 808-815, 2012.
18. Liu ZH, Qi YC, Pan PG, Ma MM, Qian XG and Fu WJ: Clinical observation on treatment of clearing the governor vessel and refreshing the mind needling in neural development and remediation of children with cerebral palsy. Chin J Integr Med 19: 505-509, 2013.

19. Wang K, Zhang R, He F, Lin LB, Xiang XH, Ping XJ, Han JS, Zhao GP, Zhang QH and Cui CL: Electroacupuncture frequency-related transcriptional response in rat arcuate nucleus revealed region-distinctive changes in response to low- and high-frequency electroacupuncture. J Neurosci Res 90: 1464-1473, 2012.

20. Kim YR, Kim HN, Ahn SM, Choi YH, Shin HK and Choi BT: Electroacupuncture promotes post-stroke functional recovery via enhancing endogenous neurogenesis in mouse focal cerebral ischemia. PLoS One 9: e90000, 2014.

21. Sharma A, Sane H, Kulkarni P, D'sa M, Gokulchandran N and Badhe P: Improved quality of life in a case of cerebral palsy after bone marrow mononuclear cell transplantation. Cell J 17: 389-394, 2015.

22. Porambo M, Phillips AW, Marx J, Ternes K, Arauz E, Pletnikov M, Wilson MA, Rothstein JD, Johnston MV and Fatemi A: Transplanted glial restricted precursor cells improve neurobehavioral and neuropathological outcomes in a mouse model of neonatal white matter injury despite limited cell survival. Glia 63: 452-465, 2015.

23. Fernández-López D, Natarajan N, Ashwal S and Vexler ZS: Mechanisms of perinatal arterial ischemic stroke. J Cereb Blood Flow Metab 34: 921-932, 2014.

24. Back SA, Han BH, Luo NL, Chricton CA, Xanthoudakis S, Tam J, Arvin KL and Holtzman DM: Selective vulnerability of late oligodendrocyte progenitors to hypoxia-ischemia. J Neurosci 22: 455-463, 2002.

25. Follett PL, Deng W, Dai W, Talos DM, Massillon LJ, Rosenberg PA, Volpe JJ and Jensen FE: Glutamate receptor-mediated oligodendrocyte toxicity in periventricular leukomalacia: a protective role for topiramate. J Neurosci 24: 4412-4420, 2004.

26. Volpe JJ: Brain injury in premature infants: a complex amalgam of destructive and developmental disturbances. Lancet Neurol 8: 110-124, 2009.

27. Ploughman M: Exercise is brain food: the effects of physical activity on cognitive function. Dev Neurorehabil 11: 236-240, 2008.

28. Cengiz P, Uluc K, Kendigelen P, Akture E, Hutchinson E, Song C, Zhang L, Lee J, Budoff GE, Meyerand E, et al: Chronic neurological deficits in mice after perinatal hypoxia and ischemia correlate with hemispheric tissue loss and white matter injury detected by MRI. Dev Neurosci 33: 270-279, 2011.

29. Fan LW, Lin S, Pang Y, Lei M, Zhang F, Rhodes PG and Cai Z: Hypoxia-ischemia induced neurological dysfunction and brain injury in the neonatal rat. Behav Brain Res 165: 80-90, 2005.

30. Zhang ZG and Chopp M: Neurorestorative therapies for stroke: underlying mechanisms and translation to the clinic. Lancet Neurol 8: 491-500, 2009.

31. Abe K, Yamashita T, Takizawa S, Kuroda S, Kinouchi H and Kawahara N: Stem cell therapy for cerebral ischemia: from basic science to clinical applications. J Cereb Blood Flow Metab 32: 1317-1331, 2012.

32. Shinoyama M, Ideguchi M, Kida H, Kajiwara K, Kagawa Y, Maeda Y, Nomura S and Suzuki M: Cortical region-specific engraftment of embryonic stem cell-derived neural progenitor cells restores axonal sprouting to a subcortical target and achieves motor functional recovery in a mouse model of neonatal hypoxic-ischemic brain injury. Front Cell Neurosci 7: $128,2013$.

33. Rice JE III, Vannucci RC and Brierley JB: The influence of immaturity on hypoxic-ischemic brain damage in the rat. Ann Neurol 9: 131-141, 1981

34. Duarte NA, Grecco LA, Galli M, Fregni F and Oliveira CS: Effect of transcranial direct-current stimulation combined with treadmill training on balance and functional performance in children with cerebral palsy: a double-blind randomized controlled trial. PLoS One 9: e105777, 2014.

35. Yager JY: Animal models of hypoxic-ischemic brain damage in the newborn. Semin Pediatr Neurol 11: 31-46, 2004.

36. Towfighi J, Zec N, Yager J, Housman C and Vannucci RC: Temporal evolution of neuropathologic changes in an immature rat model of cerebral hypoxia: a light microscopic study. Acta Neuropathol 90: 375-386, 1995. 
37. McPherson RJ, Mascher-Denen M and Juul SE: Postnatal stress produces hyperglycemia in adult rats exposed to hypoxia-ischemia. Pediatr Res 66: 278-282, 2009.

38. Jablonska B, Scafidi J, Aguirre A, Vaccarino F, Nguyen V, Borok E, Horvath TL, Rowitch DH and Gallo V: Oligodendrocyte regeneration after neonatal hypoxia requires FoxO1-mediated p2 $7^{\text {Kipl }}$ expression. J Neurosci 32: 14775-14793, 2012.

39. Couillard-Despres S, Winner B, Schaubeck S, Aigner R, Vroemen M, Weidner N, Bogdahn U, Winkler J, Kuhn HG and Aigner L: Doublecortin expression levels in adult brain reflect neurogenesis. Eur J Neurosci 21: 1-14, 2005.

40. Schinder AF and Poo M: The neurotrophin hypothesis for synaptic plasticity. Trends Neurosci 23: 639-645, 2000.

41. Ding Y, Li J, Luan X, Ding YH, Lai Q, Rafols JA, Phillis JW, Clark JC and Diaz FG: Exercise pre-conditioning reduces brain damage in ischemic rats that may be associated with regional angiogenesis and cellular overexpression of neurotrophin Neuroscience 124: 583-591, 2004.

42. Hwang DH, Shin HY, Kwon MJ, Choi JY, Ryu BY and Kim BG: Survival of neural stem cell grafts in the lesioned spinal cord is enhanced by a combination of treadmill locomotor training via insulin-like growth factor-1 signaling. J Neurosci 34: 12788-12800, 2014

43. Skup M, Dwornik A, Macias M, Sulejczak D, Wiater M and Czarkowska-Bauch J: Long-term locomotor training up-regulates $\operatorname{TrkB}(\mathrm{FL})$ receptor-like proteins, brain-derived neurotrophic factor, and neurotrophin 4 with different topographies of expression in oligodendroglia and neurons in the spinal cord. Exp Neurol 176: 289-307, 2002.

44. Ying Z, Roy RR, Zhong H, Zdunowski S, Edgerton VR and Gomez-Pinilla F: BDNF-exercise interactions in the recovery of symmetrical stepping after a cervical hemisection in rats Neuroscience 155: 1070-1078, 2008.

45. Manni L, Albanesi M, Guaragna M, Barbaro Paparo S and Aloe L: Neurotrophins and acupuncture. Auton Neurosci 157: 9-17, 2010.
46. Sun Y, Jin K, Childs JT, Xie L, Mao XO and Greenberg DA: Vascular endothelial growth factor-B (VEGFB) stimulates neurogenesis: evidence from knockout mice and growth factor administration. Dev Biol 289: 329-335, 2006.

47. Ding Y, Zhang RY, He B, Liu Z, Zhang K, Ruan JW, Ling EA, Wu JL and Zeng YS: Combination of electroacupuncture and grafted mesenchymal stem cells overexpressing TrkC improves remyelination and function in demyelinated spinal cord of rats. Sci Rep 5: 9133, 2015.

48. Strube W, Nitsche MA, Wobrock T, Bunse T, Rein B, Herrmann M, Schmitt A, Nieratschker V, Witt SH, Rietschel M, et al: BDNF-Val66Met-polymorphism impact on cortical plasticity in schizophrenia patients: a proof-of-concept study. Int J Neuropsychopharmacol 18: 18, 2014.

49. Ohira K, Furuta T, Hioki H, Nakamura KC, Kuramoto E, Tanaka Y, Funatsu N, Shimizu K, Oishi T, Hayashi M, et al: Ischemia-induced neurogenesis of neocortical layer 1 progenitor cells. Nat Neurosci 13: 173-179, 2010.

50. Johnston MV: Plasticity in the developing brain: implications for rehabilitation. Dev Disabil Res Rev 15: 94-101, 2009.

51. Huang SF, Ding Y, Ruan JW, Zhang W, Wu JL, He B, Zhang YJ, Li Y and Zeng YS: An experimental electro-acupuncture study in treatment of the rat demyelinated spinal cord injury induced by ethidium bromide. Neurosci Res 70: 294-304, 2011

52. Elfving B, Christensen T, Ratner C, Wienecke J and Klein AB Transient activation of mTOR following forced treadmill exercise in rats. Synapse 67: 620-625, 2013.

53. Kim JY, Kim JM and Ko EY: The effect of the action observation physical training on the upper extremity function in children with cerebral palsy. J Exerc Rehabil 10: 176-183, 2014.

54. Kwon SJ, Song KS, Kim H, Kim YS, Choi WS and Kwon SO: Low-intensity treadmill exercise and bright light upregulate brain-derived neurotrophic factor expression and intracellular signaling pathway in rat hippocampus and cerebral cortex. Anim Cells Syst 18: 135-142, 2014. 